\title{
SPARC suppresses lymph node metastasis by regulating the expression of VEGFs in ovarian carcinoma
}

\author{
FENGHUI PENG ${ }^{1 *}$, YANPING ZHONG ${ }^{2 *}$, YUNFENG LIU $^{1}$, YUEMING ZHANG ${ }^{2}$, \\ YIHONG XIE ${ }^{1}$, YINGXIN LU ${ }^{1}$, XINYIN ZHANG ${ }^{1}$ and DANRONG LI ${ }^{1,2}$ \\ ${ }^{1}$ Affliated Tumor Hospital of Guangxi Medical University, ${ }^{2}$ Medical Scientific Research Center, \\ Guangxi Medical University, Nanning, Guangxi Zhuang Autonomous Region 530021, P.R. China
}

Received July 30, 2017; Accepted October 12, 2017

DOI: 10.3892/ijo.2017.4168

\begin{abstract}
Lymph node metastasis is one of the most valuable determinants for the prognosis of ovarian cancer. However, the molecular mechanisms underlying lymphangiogenesis in ovarian cancer is still poorly understood. Secreted protein acidic and rich in cysteine (SPARC), a $\mathrm{Ca}^{2+}$-binding matricellular glycoprotein that modulates cell adhesion, migration and differentiation, is thought to play a decisive role in tumor metastasis. Vascular endothelial growth factor (VEGF)-C and VEGF-D contributes to tumor-associated lymphatic vessel growth, enhancing the metastatic spread of tumor cells to lymph nodes. The aim of the present study was to investigate the relationship among SPARC, VEGFs and lymph node metastasis in ovarian cancer. We found that SKOV3 cells expressed high-level SPARC, much more than SKOV3-PM4 cells (a subline with high directional lymphatic metastatic potentials established from the metastatic lymph node generated by human ovarian carcinoma cell line SKOV3 in nude mice) did at both mRNA and protein levels. A SPARCoverexpressed SKOV3-PM4 cell line was constructed and it was found that upregulation of SPARC expression suppressed the growth, migration and invasion of SKOV3-PM4 cells as well as markedly reduced the expression of VEGF-D at both mRNA and protein level by quantitative real-time polymerase chain reaction (qRT-PCR) and western blot assay. In 47 of ovarian malignant tissues, the expression of SPARC, VEGF-C and VEGF-D were determined by immunohistochemistry. Lymphatic microvessel density (LVD) and microvessel density (MVD) were evaluated by immunostaining with CD34 and D2-40 antibodies, respectively. We found that SPARC expression was significantly lower in tissues with lymph node metastasis as compared to tissues without lymph node metastasis. SPARC expression was inversely
\end{abstract}

Correspondence to: Dr Danrong Li, Department of Basic Research, Guangxi Institute for Cancer Research, 71 Hedi Road, Nanning, Guangxi Zhuang Autonomous Region 530021, P.R. China E-mail: danrongli@163.com

${ }^{*}$ Contributed equally

Key words: ovarian carcinoma, lymph node metastasis, secreted protein acidic and rich in cysteine, vascular endothelial growth factor, angiogenesis, lymphangiogenesis associated with the degree of malignancy and it had a negative correlation with VEGF-C expression, VEGF-D expression, LVD and MVD which were actually higher for advanced tumors than for non-advanced tumors. These results suggest SPARC might function as a tumor suppressor inhibiting angiogenesis and lymphangiogenesis in ovarian cancer by reducing the expression of VEGF-C and VEGF-D.

\section{Introduction}

It is generally known that ovarian cancer is one of the leading causes of cancer deaths in women and the second most common gynecologic cancer (1). Metastasis, which is mainly responsible for the high mortality of ovarian cancer, includes direct spread, lymph node metastasis, implantation metastasis and hematogenous metastasis. Of the several ways of metastasis, lymph node metastasis is one of the most valuable indicators of biological characteristics of patients (2). Patients with positive lymph node have a significantly shorter overall survival than node-negative patients. Although the combined treatments of surgery, radiotherapy, chemotherapy and endocrine therapy have greatly improved the survival rate of ovarian cancer patients, the treatment effect of patients with relapse or metastasis is still not ideal. Molecular targeted therapy might provide a new means for those hard-to-treat ovarian cancer patients. However, due to the absence of well-defined molecular targets, more extensive genomic and biological studies of ovarian cancer are required to better understand the complexity of the disease and to develop effective treatments.

SKOV3-PM4, a subline of human ovarian carcinoma cell line with high directional lymphatic metastasis, was screened and established after repeated in vivo passage with nude mice and its characteristics were compared with the parental SKOV3 line by our research group. An isobaric tag for relative and absolute quantitation labelling followed by nano liquid chromatography-matrix-assisted laser desorption ionizationtime of flight-tandem mass spectrometry was used to identify the differential expression proteins between SKOV3 cell line and SKOV3-PM4 cell line. Bioinformatics analysis revealed that downregulation of SPARC is closely related to lymph node metastasis in ovarian cancer $(3,4)$.

SPARC, also known as $43 \mathrm{~K}$ protein, osteonectin and BM-40, is a calcium-binding glycoprotein first reported by 
Table I. qRT-PCR primers.

\begin{tabular}{|c|c|c|}
\hline Gene & Primer sequence & Annealing temperature $\left({ }^{\circ} \mathrm{C}\right)$ \\
\hline GAPDH & $\begin{array}{l}\text { Forward: 5'-GTCAAGGCTGAGAACGGGAA-3' } \\
\text { Reverse: 5'-AAATGAGCCCCAGCCTTCTC-3' }\end{array}$ & 60 \\
\hline SPARC & $\begin{array}{l}\text { Forward: 5'-GCAGCAATGACAACAAGACCT-3' } \\
\text { Reverse: 5'-ATTCGGTCAGCTCAGAGTCCA-3' }\end{array}$ & 60 \\
\hline VEGF-A & $\begin{array}{l}\text { Forward: 5'-AGGAGGGCAGAATCATCA-3' } \\
\text { Reverse: 5'-AGATGTCCACCAGGGTCTC-3' }\end{array}$ & 62 \\
\hline VEGF-B & $\begin{array}{l}\text { Forward: 5'-GTACCCGAGCAGTCAGCT-3' } \\
\text { Reverse: 5'-CCCTGTCTGGCTTCACAG-3' }\end{array}$ & 62 \\
\hline VEGF-C & $\begin{array}{l}\text { Forward: 5'-ACAGGCCAACCTCAACTCAA-3' } \\
\text { Reverse: 5'-GTAGACGGACACACATGGAG-3' }\end{array}$ & 62 \\
\hline VEGF-D & $\begin{array}{l}\text { Forward: 5'-TCCCATCGGTCCACTAGGTT-3' } \\
\text { Reverse: 5'-TGGTACTCTTCCCCAGCTCA-3' }\end{array}$ & 62 \\
\hline VEGF-E & $\begin{array}{l}\text { Forward: 5'-ATTCACAGCCCAAGGTTT-3' } \\
\text { Reverse: 5'-AGCCCAAATCTTTCATCAA-3' }\end{array}$ & 61 \\
\hline
\end{tabular}

Sage and his colleagues in 1984 (5). SPARC is involved in cell renewal, embryonic development, angiogenesis, tissue remodeling and modulation of cell-matrix interactions (6). Growing evidence suggests that SPARC abnormally expresses and plays an important role in a variety of cancers (7-10). VEGFs, mainly including VEGF-A, VEGF-B, VEGF-C, VEGF-D and VEGF-E family members, considered as the most effective pro-angiogenic factors secreted by tumor cells, also play an vital role in tumor development, invasion and metastases formation. A number of reports have shown that SPARC inhibits VEGF expression during the formation of new blood vessel by which indirectly restrain the development, growth, invasion and metastasis of tumor cells (11-13).

Despite these advances, the relationship among SPARC, VEGFs and lymph node metastasis in ovarian cancer has not been completely investigated. In the present study, we increased the expression of SPARC in SKOV3-PM4 cells by lentivirus-mediated RNA overexpression, and then a series of experiments were performed in vitro to clarify the role of SPARC in lymph node metastasis of ovarian cancer. We found that overexpression of SPARC could obviously attenuate SKOV3-PM4 cell proliferation, migration and invasion. In addition, the expression of SPARC, VEGFs, D2-40 (vascular lymphatic marker) and CD34 (vascular endothelial marker) in human ovarian malignant tumor tissue specimens were measured by immunohistochemistry. The numbers of lymphatic microvessel and microvessel formation were counted by D2-40 and CD34 antigen staining, respectively. Our results showed that SPARC inhibited the ovarian cancer metastasis of lymph nodes by decreasing the expression level of VEGF-C and VEGF-D.

\section{Materials and methods}

Cell line and plasmids. SKOV3 cell line was purchased from the Cell Resources Center of Shanghai Biological Sciences Institute. SKOV3-PM4 cell line was established by our research group (3) and preserved at Medical Scientific Research Center of Guangxi Medical University. HEK293T cells (Human embryonic kidney cells expressing the large simian virus 40 $\mathrm{T}$ antigen), Plasmid H1, Plasmid $\mathrm{H} 2$ and $\mathrm{DH} 5 \alpha$ E. coli were obtained from the Oncology Laboratory of Medical Scientific Research Center of Guangxi Medical University.

Construction of the SPARC overexpression lentiviral vector and virus packaging. To construct a plasmid expressing SPARC, the sequence was amplifed with the primers (Table I) and they both contained $M l u \mathrm{I}$ and XhoI restriction enzyme sites. Polymerase chain rection (PCR) was used to clone the SPARC gene using the following reaction conditions: $95^{\circ} \mathrm{C}$ pre-denaturation for $5 \mathrm{~min}, 94^{\circ} \mathrm{C}$ denaturation for $30 \mathrm{sec}, 57^{\circ} \mathrm{C}$ annealing for $30 \mathrm{sec}, 72^{\circ} \mathrm{C}$ extension for $30 \mathrm{sec}$ for 30 cycles, $72^{\circ} \mathrm{C}$ extension for $10 \mathrm{~min}$. The PCR products were detected by a $1 \%$ agarose gel electrophoresis and were sequenced by the Beijing Genomics Institute (Shenzhen, China). The recombinant plasmids were transformed into DH5 $\alpha$ E. coli.

Cell culture and transfection. SKOV3 cells and SKOV3-PM4 cells were cultured in RPMI-1640 medium (Gibco, Gaithersburg, MD, USA), supplemented with $10 \%$ fetal bovine serum (FBS; Gibco) in a humidified incubator and $1 \%$ penicillin/streptomycin at $37^{\circ} \mathrm{C}$ with $5 \% \mathrm{CO}_{2}$ atmosphere. SKOV3-PM4 cells were cultured in 6-well tissue culture plates and were infected with lentivirus for $24 \mathrm{~h}$. Then, the medium was replaced with fresh complete medium and cultured for $48 \mathrm{~h}$, followed by selection with puromycin $(2 \mu \mathrm{g} / \mathrm{ml})$. Cells were observed under fluorescence microscope to confirm that $>80 \%$ of cells were GFP-positive and the SPARC expression in the stably transfected cells were examined by real-time PCR and western blotting. The tranfected cells in the present study were divided into 2 groups: group 1 (SKOV3-PM4-NC, cells transfected with empty vectors), and group 2 (SKOV3PM4-SPARC, cells transfected with SPARC overexpressed RNA). 
qRT-PCR for SPARC and VEGFs mRNA expression. Total RNA of SPARC and VEGFs were isolated from cultured cell lines using the TRIzol reagent (Invitrogen, Carlsbad, CA, USA) according to the instructions of the manufacturer. The primers (Table I) were designed by Oligo 7.0 software and synthesized by Takara Biotechnology Co. Ltd. (Dalian, China). The mRNA level of the target gene was quantified by measuring the $\mathrm{CT}$ value to determine its relative expression. The results are reported using the fold change in the gene expression of the target genes relative to the internal control gene (GAPDH). The mean-fold change in target gene expression was calculated as $2^{-\Delta \Delta \mathrm{CT}}$, where $\Delta \Delta \mathrm{CT}=\left(\mathrm{CT}_{\text {Target }}-\mathrm{CT}_{\mathrm{GAPDH}}\right)$ sample $-\left(\mathrm{CT}_{\text {Target }}-\mathrm{CT}_{\mathrm{GAPDH}}\right)$ control.

Western blotting for SPARC and VEGF protein expression. The protein concentration was determined using the BCA assay. One hundred micrograms of total protein was loaded into each well and seperated by electrophoresis in a $10 \%$ SDS-PAGE gel. The proteins were electro-transferred to polyvinylidene fluoride (PVDF) membranes. After blocking with 5\% non-fat milk in phosphate-buffered saline (PBS) containing 0.5\% Tween-20 (PBST), the PVDF membranes were incubated overnight at $4^{\circ} \mathrm{C}$ with SPARC (1:500; Cell Signaling Technology, Danvers, MA, USA), VEGF-A (1:500; Abcam, Cambridge, MA, USA), VEGF-B (1:500; Abcam), VEGF-C (1:500; Abcam), VEGF-D (1:500; Abcam), VEGF-E (1:500; Abcam) and GAPDH $(1: 1,000$; Abcam) primary monoclonal antibodies in PBST buffer containing $0.1 \%$ Tween-20. The next day, the membranes were washed and incubated with a secondary antibody conjugated with horseradish peroxidase (HRP) $(1: 2,000 ;$ Abcam). The intensity of protein staining was determined with ImageJ software.

Laser confocal microscopy for the distribution of SPARC protein. Laser scanning confocal microscopy was used to observe the distribution of SPARC protein in cells. Cells were seeded in the glass base of the plate at $1 \times 10^{5}$ cells $/ \mathrm{ml}$ in RPMI1640 with $10 \%$ FBS. After $24 \mathrm{~h}$, supernatants were discarded and the cells were fixed with methanol for $10 \mathrm{~min}$. After washing with PBS three times with PBS for 2 min each time, cells were incubated with a SPARC primary antibody (1:500; Cell Signaling Technology) for $2 \mathrm{~h}$ and then with fluorescence labeling secondary antibodies (1:2,000; Abcam) for $2 \mathrm{~h}$. The sections were counterstained with DAPI. Localization and expression of SPARC protein in cells were visualized and captured using laser confocal microscopy (Nikon A1; Nikon, Tokyo, Japan).

Cell counting method and colony formation test for cell proliferation. To determine the growth rate of cells after lentiviral treatments, $10^{4}$ cells were seeded in 24-well plate in $100 \mu 1$ RPMI-1640 with 10\% FBS. The cells were digested and counted under the microscopy for 7 days. Cells $\left(10^{3}\right)$ were added to 6-well plates with RPMI-1640 containing $10 \%$ FBS, and each cell group contained 3 -wells. After incubation at $37^{\circ} \mathrm{C}$ for 14 days, the cells were washed twice with PBS and were stained with Giemsa solution. The number of colonies containing $>50$ cells was counted under a microscope.
Transwell assay for cell migration and invasion. Each Transwell (BD Biosciences, Bedford, MA, USA) was coated with $100 \mu \mathrm{l}$ Matrigel (BD Biosciences). Cells were adjusted to a density of of $5 \times 10^{4}$ cells $/ \mathrm{ml}$, resuspended in $200 \mu 1$ serum-free medium and seeded into the upper chamber. Complete medium was added to the lower chamber as a chemotactic factor. After 24-h incubation, cells remaining on the upper surface were removed, and cells on the lower surface were fixed, stained with Giemsa, then counted. Cell migration assay was also performed using Transwells without Matrigel coating.

Immunohistochemistry for expression of VEGF-C, VEGF-D, LVD and MVD in tissue specimens of human ovarian cancer. Forty-seven human ovarian malignant tumor tissue specimens were collected from patients who underwent surgical treatment at the Cancer Hospital Affliated to Guangxi Medical University (Nanning, China) from January 2008 to May 2013. None of the patients received any therapies prior to surgery. Of the 47 ovarian cancer patients (mean age $45.0 \pm 3.1$ years), 32 were serous carcinoma and 15 non-serous carcinoma (including 8 mucinous cystadenocarcinoma, 5 endometrioid carcinoma and 2 clear cell carcinoma). Of all the patients, 27 had no lymph node metastasis and 20 had lymph node metastasis. All human ovarian tissue specimens were collected during the operation. Patients or their family members' consent were obtained before the collection of surgical materials and written informed consents were obtained from all the patients. This study was approved by the Ethical Review Committee of the Affliated Tumor Hospital of Guangxi Medical University (Guangxi, China).

Tissue sections $(4 \mu \mathrm{m})$ were cut, and deparaffinized. The sections were incubated overnight at $4^{\circ} \mathrm{C}$ with SPARC, VEGF-C, VEGF-D, D2-40 (1:100) and CD34 (1:100; Beijing Zhongshan Golden Bridge Biotechnology Co. Ltd., Beijing, China) primary antibody respectively and PBS was used as a negative control.

Both cytoplasm and the membrance of nucleus appeared brown-yellow were considered to be SPARC, VEGF-C and VEGF-D positive expression. For the assessment of SPARC, VEGF-C and VEGF-D staining, each tissue section was independently analyzed by two pathologists in the doubleblinded manner. According to the proportion and the intensity of positive staining, the stained cells were scored from 0 to 3 ( 0 point, $0 \%$ positive tumor cells; 1 point, $0-33.33 \%$; 2 point, $33.33-66.67 \% ; 3$ point, $66.67-100 \%)$. While the staining intensity was classified as a scale of 0 (no staining), 1 (light yellow), 2 (yellowish brown) and 3 (brown). The final staining score was calculated by adding together the percentage and intensity scores, and the scores of $0,1-3,4-6$ and $>7$ were converted into four grades negative, weak, moderate and strong, respectively.

Lymphatic microvessel and microvessels were identified by immunostaining endothelial cells with D2-40 and CD34 monoclonal antibodies, respectively. LVD and MVD were assessed according to the methods summarized by Van der Auwera et al (14). The entire section was scanned systematically at low magnification (x40) in order to identify the most intense areas of neovascularization within the tumor. The lymphatic microvessel and microvessels were counted at high magnification (x200), and the average count of three fields was calculated. 

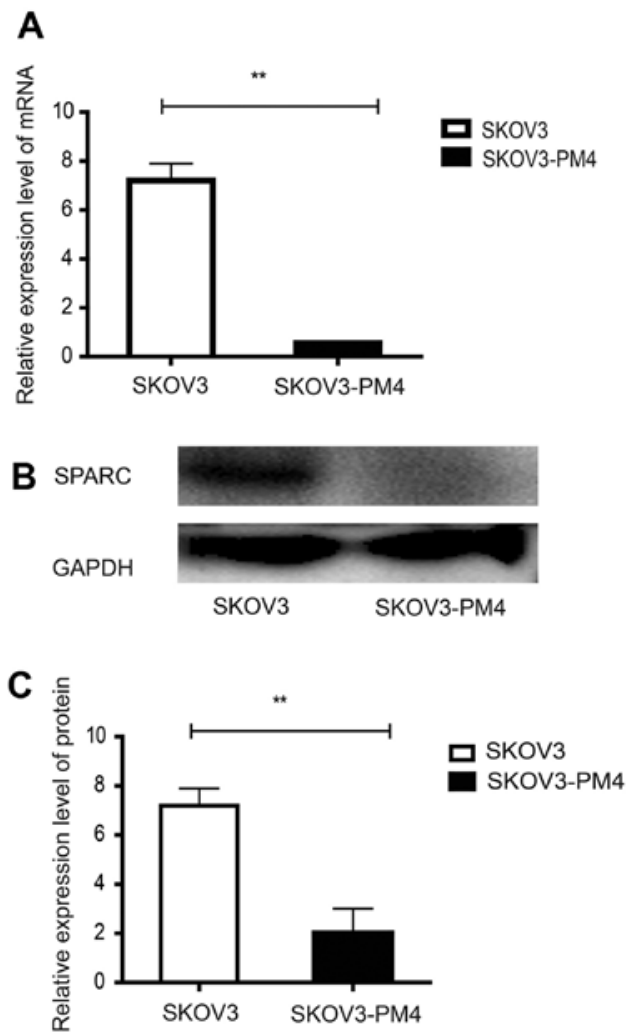

Figure 1. The expression level of SPARC in SKOV3 cells and SKOV3-PM4 cells. (A) Total RNA was isolated from the cells and SPARC mRNA expression level was measured by qRT-PCR. (B and C) The protein expression level of SPARC was measured by western blotting, GAPDH was used for internal control. Data are presented as mean $\pm \mathrm{SD}, \mathrm{n}=3\left({ }^{* *} \mathrm{P}<0.01\right)$.

Statistical analysis. The data were analyzed with SPSS 16.0 software. Enumeration data are presented as mean \pm standard deviation, the results from two groups were compared by t-test. $\chi^{2}$-test was used to analyze the clinical data. Correlation analysis of VEGF-C, VEGF-D with SPARC expression were studied using the Spearman's rank correlation test. The correlation of SPARC expression with LVD, MVD were analyzed using the Mann-Whitney $\mathrm{U}$ test. $\mathrm{P}<0.05$ were considered statistically significant.

\section{Results}

SPARC expression in SKOV3 and SKOV3-PM4 cells. To evaluate the expression of SPARC in SKOV3 cells and SKOV3-PM4 cells, qRT-PCR and western blotting were performed. As shown in Fig. 1, SKOV3-PM4 cells exhibited a significantly decreased SPARC expression in both mRNA and protein level compared to SKOV3 cells $(\mathrm{P}<0.01)$.

Construction of SPARC overexpression SKOV3-PM4 cells. To functionally link SPARC expression with lymph node metastasis, we transfected SKOV3-PM4 cells with SPARC overexpressed lentivirus vector labelled with green fluorescent protein. The transfected cells stably expressed green fluorescent protein which could be observed using an inverted fluorescence microscope.

SKOV3-PM4-SPARC cells expressed high-level SPARC, much more than SKOV3-PM4 cells and SKOV3-PM4-NC cells
A

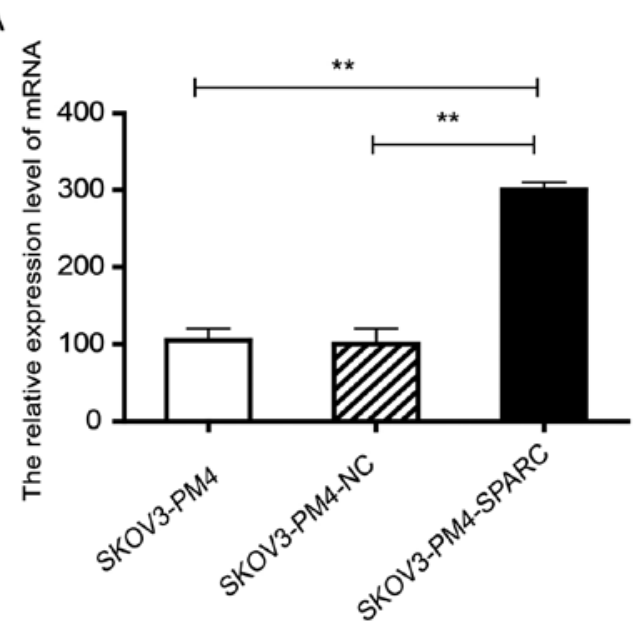

B

GAPDH

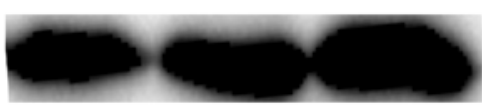

SPARC

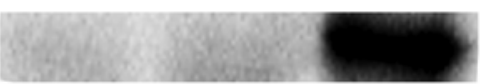

C

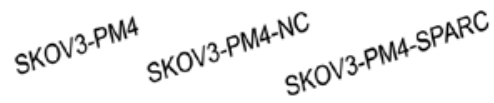

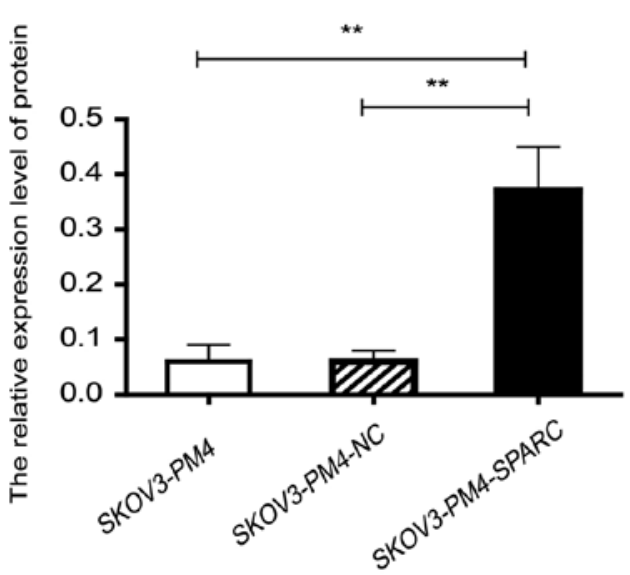

Figure 2. Verification of overexpression of SPARC in SKOV3-PM4-SPARC cells. (A) qRT-PCR analysis of SPARC mRNA expression. (B and C) Western blot analysis of SPARC protein expression. Data are presented as mean $\pm \mathrm{SD}$, $\mathrm{n}=3\left({ }^{* *} \mathrm{P}<0.01\right)$.

at both mRNA and protein levels $(\mathrm{P}<0.01 ;$ Fig. 2$)$ The results showed that SPARC gene was successfully overexpressed in SKOV3-PM4 cells.

Proliferation of SKOV3-PM4 cells. The effect of overexpressed SPARC on the proliferation of SKOV3-PM4 cells was investigated by soft agar colony formation assay and cell proliferation assay. The ability of colony formation of SKOV3-PM4-SPARC cells was markedly lower than that of SKOV3-PM4 and SKOV3-PM4-NC cells $(\mathrm{P}<0.05$; Fig. 3A-C). Differences of cell proliferation were observed among SKOV3-PM4, SKOV3-PM4-NC and SKOV3-PM4SPARC cells from the second day to the seventh day after cells seeding. The doubling time of cells was $37.20 \pm 1.87$, $37.19 \pm 1.83$ and $44.54 \pm 2.89 \mathrm{~h}$, respectively $(\mathrm{P}<0.05)$. The 
A

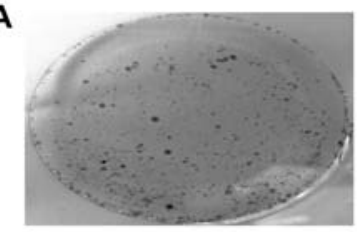

B

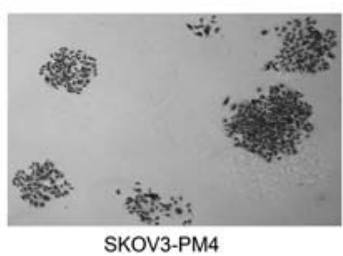

SKOV3-PM4

\section{C}

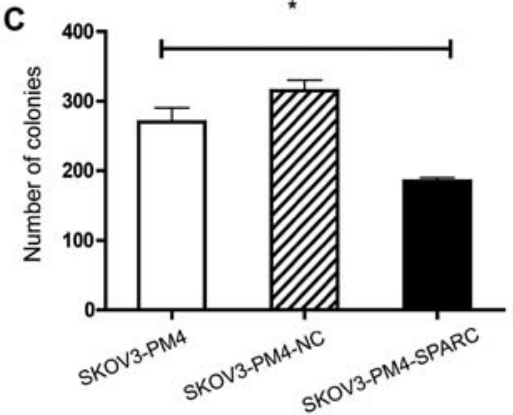

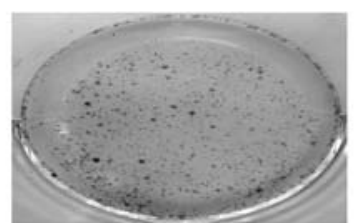
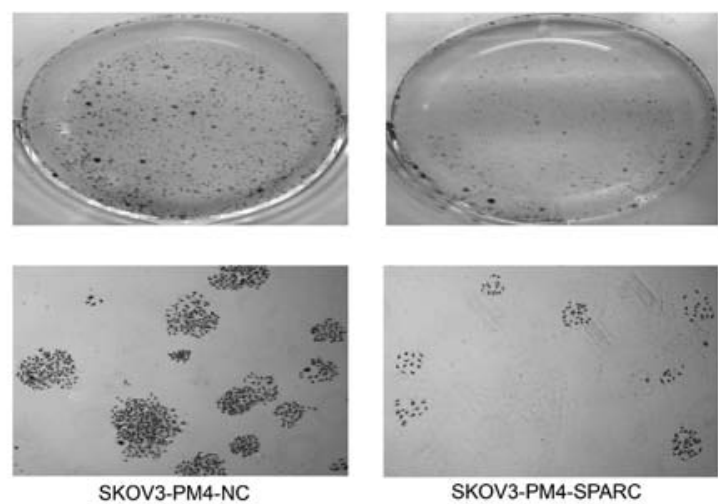

SKOV3-PM4-SPARC

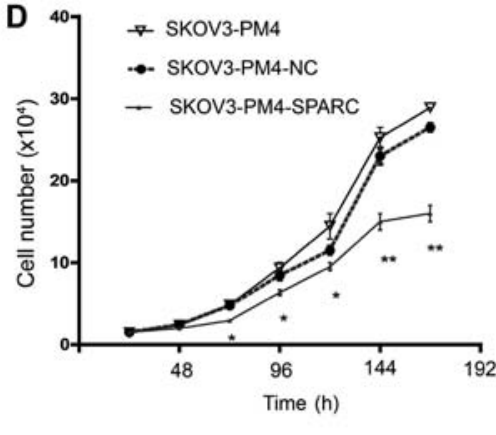

Figure 3. Overexpression of SPARC decreases the proliferation of SKOV3-PM4 cells. (A-C) The ability of colony formation of cells. Original magnification, $\mathrm{x} 40$. (D) Cell growth curves of cells. Upregulation of SPARC expression signifcantly reduced SKOV3-PM4 proliferation. Data are presented as mean \pm SD $(\mathrm{n}=3)\left({ }^{*} \mathrm{P}<0.05,{ }^{* *} \mathrm{P}<0.01\right)$.
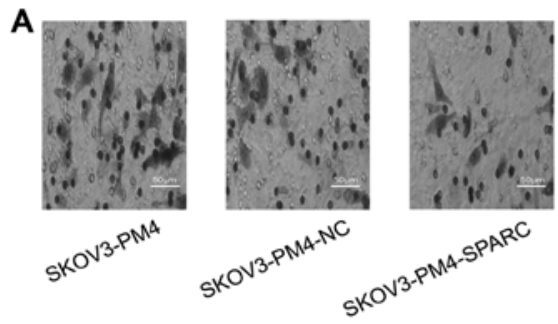

C

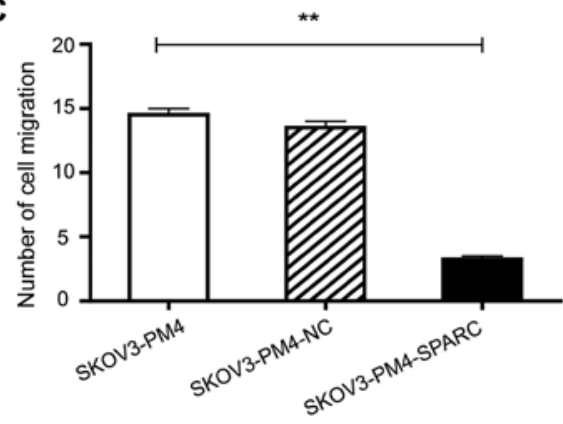

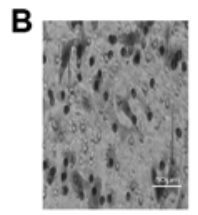
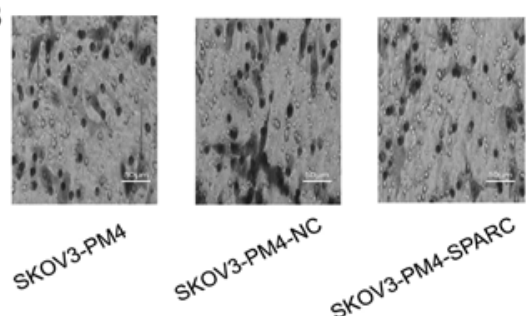

D

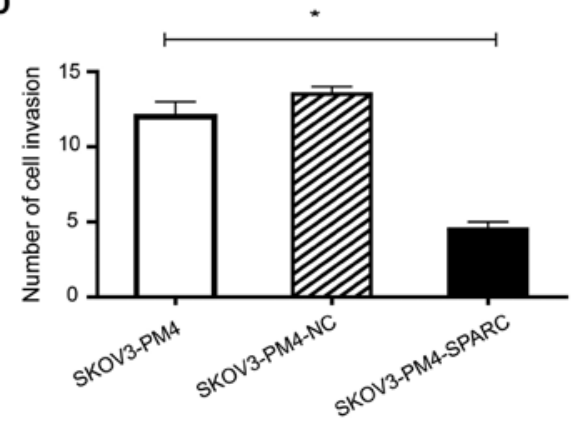

Figure 4. SPARC overexpression suppressed migration and invasion of SKOV3-PM4 cells. (A and C) The migration ability of SKOV3-PM4 cells was determined using Transwells assay without Matrigel coating. (B and D) The invasion of SKOV3-PM4 cells was determined using the Matrigel-based Transwell assay. Cells that invaded the lower surface of the membrane were fixed and stained by Giemsa. The relative number of invaded cells were observed at least 5 fields. Data are presented by at least three independent experiment and was expressed as mean $\pm \mathrm{SD} .\left({ }^{*} \mathrm{P}<0.05,{ }^{* * *} \mathrm{P}<0.01\right)$. Original magnification, $\mathrm{x} 200$. Scale bar, $50 \mu \mathrm{m}$.

growth curves of cells showed that overexpression of SPARC significantly reduced the proliferation of SKOV3-PM4 cells (Fig. 3D).

Migration and invasion of SKOV3-PM4 cells. To investigate the effects of SPARC on migration and invasion of
SKOV3-PM4 cells, Transwell assay without Matrigel and Transwell invasion assay based on Matrigel were performed by using the same cell number. The results showed that the migration (Fig. 4A and C) and invasion (Fig. 4B and D) ability of SKOV3-PM4-SPARC cells was significantly inferior to that of SKOV3-PM4 and SKOV3-PM4-NC cells. 
Table II. The relationship among SPARC, VEGF-C and VEGF-D expression in ovarian malignant tumor tissues with clinicopathological parameters.

\begin{tabular}{|c|c|c|c|c|c|c|c|}
\hline Parameters & $\mathrm{n}$ & $\begin{array}{c}\text { SPARC positive } \\
\text { rate }(\%)\end{array}$ & P-value & $\begin{array}{c}\text { VEGF-C positive } \\
\text { rate }(\%)\end{array}$ & P-value & $\begin{array}{c}\text { VEGF-D positive } \\
\text { rate }(\%)\end{array}$ & P-value \\
\hline \multicolumn{8}{|c|}{ FIGO staging } \\
\hline I-II & 9 & $9(100)$ & $0.000^{\mathrm{c}}$ & $4(44.4)$ & $0.013^{\mathrm{a}}$ & $2(22.2)$ & $0.026^{\mathrm{a}}$ \\
\hline III-IV & 38 & $7(18.4)$ & & $33(86.8)$ & & $25(65.8)$ & \\
\hline \multicolumn{8}{|c|}{ Lymph node metastasis } \\
\hline Yes & 20 & $3(15.0)$ & $0.039^{\mathrm{a}}$ & $20(100)$ & $0.003^{\mathrm{b}}$ & $15(75.0)$ & $0.027^{\mathrm{a}}$ \\
\hline No & 27 & $13(48.2)$ & & $17(62.9)$ & & $12(44.4)$ & \\
\hline
\end{tabular}

${ }^{\mathrm{a}} \mathrm{P}<0.05,{ }^{\mathrm{b}} \mathrm{P}<0.01,{ }^{\mathrm{c}} \mathrm{P}<0.001$.

A

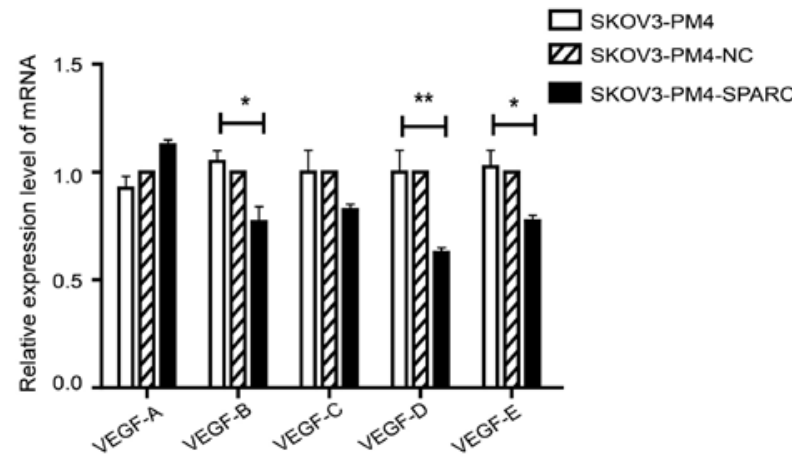

B

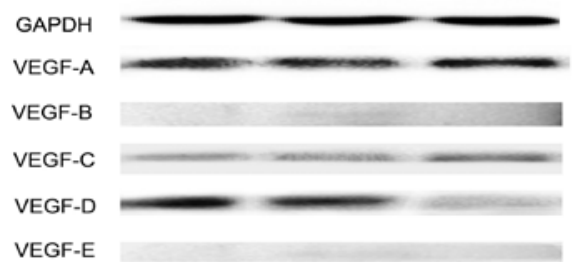

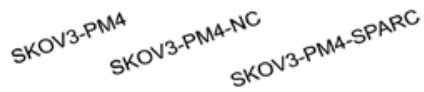

C

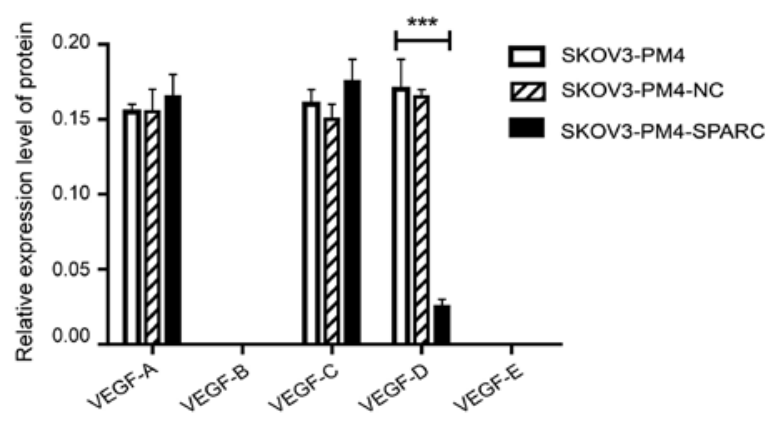

Figure 5. SPARC overexpression affects the expression level of VEGF family. (A) Total RNA was isolated from the cells and mRNA expression level of VEGF-A, VEGF-B, VEGF-C, VEGF-D and VEGF-E were examined by qRT-PCR. The experiment was repeated three times. (B and C) Protein expression level of VEGF-A, VEGF-B, VEGF-C, VEGF-D and VEGF-E were measured by western blotting using the GAPDH as loading control. Data are presented by mean $\pm \mathrm{SD}, \mathrm{n}=3 .\left({ }^{*} \mathrm{P}<0.05,{ }^{* *} \mathrm{P}<0.01,{ }^{* * *} \mathrm{P}<0.001\right)$.

The relationship between SPARC overexpression and VEGF family. To determine the relationship between SPARC overexpression and VEGF family, the expression level of VEGF family

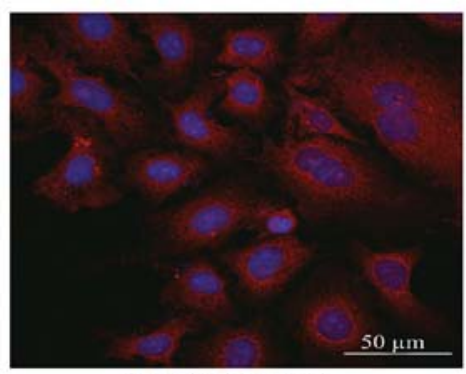

SKOV3-PM4

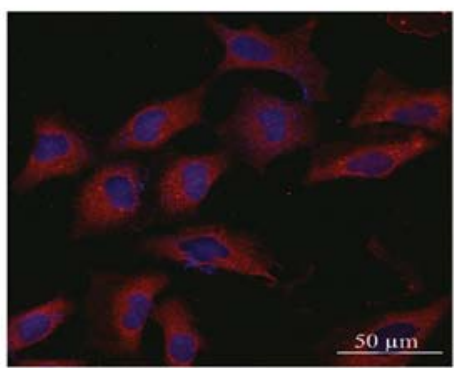

SKOV3-PM4-NC

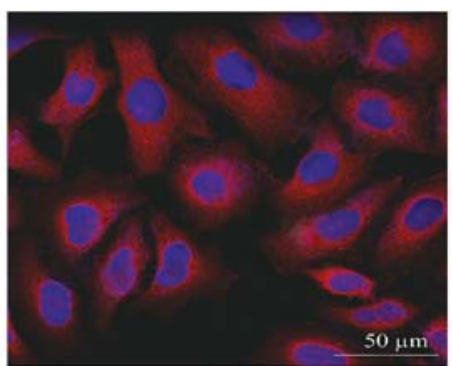

SKOV3-PM4-SPARC

Figure 6. The SPARC protein distribution detected by laser confocal microscopy. SPARC protein in SKOV3-PM4, SKOV3-PM4-NC and SKOV3PM4-SPARC cells. The nuclei are stained blue by DAPI and SPARC protein are indicated in red. The images are presentation of three reduplicate experiments. Original magnification, x600; Scale bar, $50 \mu \mathrm{m}$.

was evaluated by qRT-PCR and western blotting. As compared with SKOV3-PM4 cells, the expression level of VEGF-D in SKOV3-PM4-SPARC cells was markedly decreased while VEGF-A and VEGF-C had no significant change at either mRNA or protein level. VEGF-B and VEGF-E protein were not deteced in either SKOV3-PM4 or SKOV3-PM4-SPARC 
Table III. Analysis of the correlation among LVD, MVD and SPARC expression.

\begin{tabular}{lccccr}
\hline Groups & $\mathrm{n}$ & $\begin{array}{c}\text { LVD }(\mathrm{D} 2-40) \\
\text { mean } \pm \mathrm{SD}\end{array}$ & P-value & $\begin{array}{c}\text { MVD (CD34) } \\
\text { mean } \pm \text { SD }\end{array}$ & P-value \\
\hline SPARC positive & 16 & $11.06 \pm 2.02$ & 0.012 & $15.44 \pm 1.59$ & 0.000 \\
SPARC negative & 31 & $12.7 \pm 1.77$ & & $17.71 \pm 1.88$ & \\
\hline
\end{tabular}
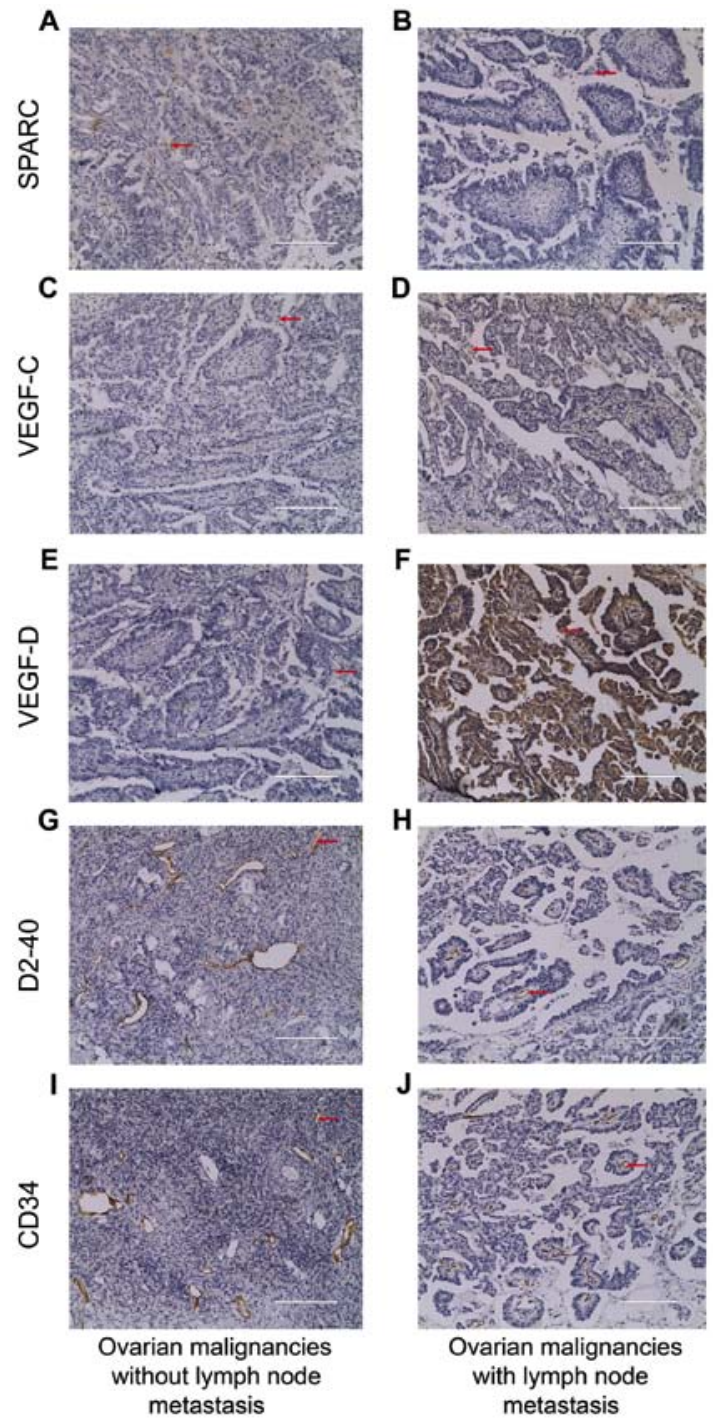

Figure 7. Expression of SPARC and the members of VEGF family were determined by immunohistochemistry. Ovarian malignant tumor tissue was processed for immunohistochemistry and the immunoreactive staining of SPARC (A and B), VEGF-C (C and D), VEGF-D (E and F), D2-40 (G and H) and $\mathrm{CD} 34$ (I and $\mathrm{J}$ ) are shown above as arrows indicate. Original magnification, $\mathrm{x} 200$. Scale bar, $200 \mu \mathrm{m}$.

cells whereas their mRNA expression level showed significant differences between these two cell lines (Fig. 5).

Distribution of SPARC protein were observed by laser confocal microscopy. Distribution of SPARC protein in SKOV3-PM4, SKOV3-PM4-NC and SKOV3-PM4-SPARC cells were observed under laser confocal microscopy. SPARC proteins are mainly distributed in cytoplasm region and membrane of the nucleus (Fig. 6).
Expression of SPARC protein and VEGF family in ovarian malignant tumor tissue. To evaluate the role of SPARC in lymph node metastasis in ovarian cancer, we carried out immunohistochemistry for SPARC, VEGF-C and VEGF-D in paraffin-embedded tissue sections obtained from 47 cases of human ovarian malignant tumors. Angiogenesis and lymphangiogenesis were evaluated by immunostaining with CD34 and D2-40 antibodies. Consistent with the result observed by laser confocal microscopy, SPARC expression was found not only in the cytoplasm but also in the membrane of the nucleus of ovarian cancer cells (Fig. 7A and B). Localization of VEGF-C and VEGF-D expression were similar to SPARC (Fig. 7C-F). The positive staining of CD34 and D2-40 was brown-yellow, localized in the membrane of vascular and lymphatic endothelial cells, respectively (Fig. 7G-J).

There was a significantly difference between the expression of SPARC, VEGF-C and VEGF-D in the lymph node-involved group compared to the lymph node non-involved group. As shown in Table II, SPARC expression was significantly lower in tissues with lymph node metastasis as compared to tissues without lymph node metastasis, and was inversly associated with the degree of malignancy. The expression of VEGF-C and VEGF-D showed the opposite results. A negative correlation was found between the expression of SPARC and VEGF-C $(\mathrm{r}=-0.394, \mathrm{P}=0.006)$ or VEGF-D $(\mathrm{r}=-0.337, \mathrm{P}=0.021)$. LVD and MVD were significantly higher in SPARC negative specimens than positive specimens using Mann-Whitney $\mathrm{U}$ test $(\mathrm{P}<0.05)$ (Table III).

\section{Discussion}

Tumor metastasis to regional lymph nodes is often regarded as the first step of tumor dissemination, which precedes the metastasis of the vascular system and is seen as a major prognostic indicator of tumor progression. SPARC is a calciumbinding protein that not only competitively binds to the cell membrane surface growth factor receptor but also binds to several residents of ECM to influence cell proliferation, adhesion, migration, matrix degradation and angiogenesis $(15,16)$. Studies have shown that SPARC plays different roles in many types of tumors including ovarian cancer and SPARC either lowly or highly expressed in ovarian cancer (17-19). Nevertheless, the specific contribution of SPARC in growth and progression of ovarian cancer is largely unexplored.

The aim of the present study was to demonstrate that SPARC might be an optional target for suppressing the lymph node metastasis in ovarian cancer. In accordance with the study by Zhang et al (12), our results showed that SPARC inhibited ovarian cancer growth in vitro as well, upregulation of SPARC expression suppressed the proliferation, migration 
and invasion of SKOV3-PM4 cells. In ovarian malignant tissues with lymph node metastasis, the expression of SPARC was significantly lower as compared to tissues without lymph node metastasis. SKOV3 cells expressed high-level SPARC, much more than SKOV3-PM4 at both mRNA and protein levels. These findings suggested that SPARC might be a crucial factor for preventing ovarian cancer lymph node metastasis. However, the underlying mechanisms of the SPARC involved in ovarian cancer progression have not been clarified.

The metastasis of ovarian cancer is a multifactorial and multistep process in which angiogenesis and lymphangiogenesis are prerequisites. Angiogenesis, in which new blood vessels develop through endothelial cell proliferation from extant vasculature, provides an effcient way for tumor cells to leave their primary site and enter the blood stream (20). Lymphangiogenesis is a complex process that has received much attention as an important mediator of tumor cell dissemination recently. It has been reported that lymphangiogenesis promotes lymphatic metastases probably by augmenting the potential entry point density of lymphatic vessels for tumor cells entering the lymphatic system (21). Increasing evidence has revealed that lymphangiogenic factors such as VEGF-C and VEGF-D induce tumor-associated lymphatic vessel growth, enhancing the metastatic spread of tumor cells to lymph nodes. Nevertheless, our knowledge of the mechanisms that underlie lymphangiogenesis still lags far behind that of angiogenesis. Therefore, further efforts are needed for better understanding of lymphatic biology to develop more effective treatments for ovarian cancer.

A differential expression of SPARC in tumor neovascular endothelial cells compared with mature vascular endothelial cells, suggesting that SPARC has been implicated in angiogenesis (22). SPARC was thought to be a key factor that inhibits the activity of VEGFs, platelet derived growth factor (PDGF), and basic fibroblast growth factor (bFGF). Studies reported that the expression of VEGFs highly increased in colon cancer along with the decreased expression of SPARC (23), and VEGF-D induced tumor lymphangiogenesis and increase the lymphatic metastasis in ovarian cancer (24). Through suppressing the expression and secretion of VEGFs, SPARC inhibited glioma growth by reducing tumor vascularity. In ovarian cancer animal models, the absence of SPARC led to high expression of VEGF, MMP2 and MMP9, indicating that SPARC modulates lymph node metastasis and promotes the metastatic potential of ovarian cancers through angiogenesis (25).

Microvessel density (MVD) and lymphatic microvessel density (LVD) is now widely used to evaluate the angiogenesis and lymphangiogenesis of tumors. In the present study, we detected the expression level of SPARC and VEGFs in human ovarian malignancy tissues by immunohistochemistry. It was revealed that SPARC expression, which was inversely associated with the degree of malignancy, had a negative correlation with VEGF-D expression, LVD and MVD which were actually higher for advanced tumors than for non-advanced tumors. We also observed overexpression of SPARC could markedly downregulate the expression of VEGF-D at both mRNA and protein level by qRT-PCR and western blot assay, implying that repression of SPARC expression may upregulate VEGF-D expression, causing the subsequent high count of MVD and
LVD. Coincidentally, it was reported that overexpression of SPARC inhibited VEGF-mediated angiogenesis (12). Another recent study confirmed that high-level expression of VEGF-D could induce invasive and metastatic behavior of breast cancer, while knock-down of it could inhibit lymphangiogenesis and lymphatic metastasis (26).

Of note, a negative correlation was also found between the expression of SPARC and VEGF-C in human ovarian malignancy tissues, whereas in cultured cells, SPARC expression was irrelevant to VEGF-C expresssion at both protein and mRNA level. Given that SPARC plays a necessary part in cell proliferation and adhesion, it is reasonable that cell culture conditions themselves could modify the expression of SPARC. Several reports have demonstrated that overexpression of VEGF-C can promote tumor lymphangiogenesis resulting in tumor metastasis to the lymph nodes in a variety of cancers and VEGF-C is associated with tumor progression $(27,28)$. Consistent with the results of our immunohistochemistry study, numerous studies have shown that the less expression of SPARC, the more expression of VEGF-C and the more progression of tumor and vice versa (29-31). Based on the above findings, it can be speculated that SPARC might function as a tumor suppressor which inhibits angiogenesis and lymphangiogenesis in ovarian cancer by reducing the expression of VEGF-C and VEGF-D.

In summary, we have provided evidence that SPARC influences lymph node metastasis by reducing the expression level of VEGF-C and VEGF-D. On the basis of the results of this study, SPARC might become a promising new therapeutic biomarker in ovarian cancer. Targeting SPARC-mediated pathway might be a valid strategy to understand how SPARC affects tumor metastasis and progress and may result in the development of anti-angiogenesis or anti-lymphangiogenesis therapy against ovarian cancer.

\section{Acknowledgements}

The present study was supported by grants obtained from the National Natural Science Foundation of China (no. 81360502) and the Guangxi Natural Science Foundation (no. 2014GXNSFAA118225).

\section{References}

1. Jemal A, Siegel R, Xu J and Ward E: Cancer statistics, 2010. CA Cancer J Clin 60: 277-300, 2010.

2. Lengyel E: Ovarian cancer development and metastasis. Am J Pathol 177: 1053-1064, 2010.

3. Ruan HY, Li DR, Li L, Guan X and Zhang W: Establishment of human ovarian carcinoma cell lines with directional highly lymphatic metastasis and study of their biological characteristics. Zhonghua Fu Chan Ke Za Zhi 42: 482-486, 2007 (In Chinese).

4. Zhang XY, Yin FQ, Liu L, Gao T, Ruan HY, Guan X, Lu YX and Li DR: Effects of HLEC on the secreted proteins of epithelial ovarian cancer cells prone to metastasize to lymph nodes. Cancer Biol Med 10: 221-226, 2013.

5. Sage H, Johnson C and Bornstein P: Characterization of a novel serum albumin-binding glycoprotein secreted by endothelial cells in culture. J Biol Chem 259: 3993-4007, 1984.

6. Bornstein P and Sage EH: Matricellular proteins: Extracellular modulators of cell function. Curr Opin Cell Biol 14: 608-616, 2002.

7. Hsiao YH, Lien HC, Hwa HL, Kuo WH, Chang KJ and Hsieh FJ: SPARC (osteonectin) in breast tumors of different histologic types and its role in the outcome of invasive ductal carcinoma. Breast J 16: 305-308, 2010. 
8. Thomas R, True LD, Bassuk JA, Lange PH and Vessella RL: Differential expression of osteonectin/SPARC during human prostate cancer progression. Clin Cancer Res 6: 1140-1149, 2000

9. Sato T, Oshima T, Yamamoto N, Yamada T, Hasegawa S, Yukawa N, Numata K, Kunisaki C, Tanaka K, Shiozawa M, et al: Clinical significance of SPARC gene expression in patients with gastric cancer. J Surg Oncol 108: 364-368, 2013.

10. Chan SK, Griffith OL, Tai IT and Jones SJ: Meta-analysis of colorectal cancer gene expression profiling studies identifies consistently reported candidate biomarkers. Cancer Epidemiol Biomarkers Prev 17: 543-552, 2008.

11. Chlenski A, Liu S, Guerrero LJ, Yang Q, Tian Y, Salwen HR Zage P and Cohn SL: SPARC expression is associated with impaired tumor growth, inhibited angiogenesis and changes in the extracellular matrix. Int J Cancer 118: 310-316, 2006.

12. Zhang JL, Chen GW, Liu YC, Wang PY, Wang X, Wan YL, Zhu J, Gao HQ, Yin J, Wang W, et al: Secreted protein acidic and rich in cysteine (SPARC) suppresses angiogenesis by down-regulating the expression of VEGF and MMP-7 in gastric cancer. PLoS One 7: e44618, 2012

13. Yunker CK, Schultz C, Lemke N, Golembieski WA, Nelson K, Ojetebbe N, Gutierrez JA, Mikkelsen T and Rempel SA: SPARC suppresses and inversely correlates with VEGF expression and secretion in SPARC-transfected U87MG cells and tumors and in primary xenograft human gliomas. Cancer Res 66: 656-656, 2006.

14. Van der Auwera I, Cao Y, Tille JC, Pepper MS, Jackson DG, Fox SB, Harris AL, Dirix LY and Vermeulen PB: First international consensus on the methodology of lymphangiogenesis quantification in solid human tumours. Br J Cancer 95: 1611-1625, 2006.

15. Tai IT and Tang MJ: SPARC in cancer biology: Its role in cancer progression and potential for therapy. Drug Resist Updat 11: 231-246, 2008

16. Kim NI, Kim GE, Lee JS and Park MH: In phyllodes tumors of the breast expression of SPARC (osteonectin/BM40) mRNA by in situ hybridization correlates with protein expression by immunohistochemistry and is associated with tumor progression. Virchows Arch 470: 91-98, 2017.

17. Bull Phelps SL, Carbon J, Miller A, Castro-Rivera E, Arnold S, Brekken RA and Lea JS: Secreted protein acidic and rich in cysteine as a regulator of murine ovarian cancer growth and chemosensitivity. Am J Obstet Gynecol 200: 180 e1-7, 2009.

18. Socha MJ, Said N, Dai Y, Kwong J, Ramalingam P, Trieu V, Desai N, Mok SC and Motamed K: Aberrant promoter methylation of SPARC in ovarian cancer. Neoplasia 11: 126-135, 2009.

19. Chen J, Wang M, Xi B, Xue J, He D, Zhang J and Zhao Y: SPARC is a key regulator of proliferation, apoptosis and invasion in human ovarian cancer. PLoS One 7: e42413, 2012.
20. Jendraschak E and Sage EH: Regulation of angiogenesis by SPARC and angiostatin: Implications for tumor cell biology. Semin Cancer Biol 7: 139-146, 1996.

21. Nakamura Y, Yasuoka H, Tsujimoto M, Kurozumi K, Nakahara M, Nakao K and Kakudo K: Importance of lymph vessels in gastric cancer: A prognostic indicator in general and a predictor for lymph node metastasis in early stage cancer. J Clin Pathol 59: 77-82, 2006.

22. Miller JR, Moon RT, Dev G, Cadigan KM, Nusse R, Hart MJ, Santos RDL, Albert IN and Rubinfeld B: Genes expressed in human tumor endothelium. Science 289: 1197-1202, 2000.

23. Liang JF, Wang HK, Xiao H, Li N, Cheng CX, Zhao YZ, Ma YB, Gao JZ, Bai RB and Zheng HX: Relationship and prognostic significance of SPARC and VEGF protein expression in colon cancer. J Exp Clin Cancer Res 29: 71-81, 2010.

24. Du LC, Chen XC, Wang D, Wen YJ, Wang CT, Wang XM, Kan B, Wei YQ and Zhao X: VEGF-D-induced draining lymphatic enlargement and tumor lymphangiogenesis promote lymph node metastasis in a xenograft model of ovarian carcinoma. Reprod Biol Endocrinol 12: 14-24, 2014.

25. Said N, Socha MJ, Olearczyk JJ, Elmarakby AA, Imig JD and Motamed K: Normalization of the ovarian cancer microenvironment by SPARC. Mol Cancer Res 5: 1015-1030, 2007.

26. Majumder M, Tutunea-Fatan E, Xin X, Rodriguez-Torres M, Torres-Garcia J, Wiebe R, Timoshenko AV, Bhattacharjee RN, Chambers AF and Lala PK: Co-expression of $\alpha 9 \beta 1$ integrin and VEGF-D confers lymphatic metastatic ability to a human breast cancer cell line MDA-MB-468LN. PLoS One 7: e35094, 2012.

27. Hirakawa S, Brown LF, Kodama S, Paavonen K, Alitalo K and Detmar M: VEGF-C-induced lymphangiogenesis in sentinel lymph nodes promotes tumor metastasis to distant sites. Blood 109: 1010-1017, 2007.

28. Kumar B, Chile SA, Ray KB, Reddy GE, Addepalli MK, Kumar AS, Ramana V and Rajagopal V: VEGF-C differentially regulates VEGF-A expression in ocular and cancer cells; promotes angiogenesis via RhoA mediated pathway. Angiogenesis 14: 371-380, 2011.

29. Bradshaw AD, Reed MJ, Carbon JG, Pinney E, Brekken RA and Sage EH: Increased fibrovascular invasion of subcutaneous polyvinyl alcohol sponges in SPARC-null mice. Wound Repair Regen 9: 522-530, 2001.

30. Yan M, Schneider J, Gear R, Lu F, LaDow K, Warshawsky D and Heffelfinger SC: Expression of angiogenic factors is upregulated in DMBA-induced rat mammary pathologies. Pathobiology 71: 253-260, 2004

31. Yu H, Zhang S, Zhang R and Zhang L: The role of VEGF-C/D and Flt-4 in the lymphatic metastasis of early-stage invasive cervical carcinoma. J Exp Clin Cancer Res 28: 98-103, 2009. 